\title{
The amide of galacturonic acid with lysine as an immunodominant component of the lipopolysaccharide core region from Proteus penneri 42 strain*
}

\author{
Agata Palusiak ${ }^{凶}$, Anna Maciejewska², Czesław Ługowski2,3 and Antoni Różalski ${ }^{4}$ \\ 1Department of General Microbiology, Institute of Microbiology, Biotechnology and Immunology, University of Łódź, Łódź, Poland; 2Department \\ of Immunochemistry, Ludwik Hirszfeld Institute of Immunology and Experimental Therapy, Polish Academy of Sciences, Wrocław, Poland; \\ ${ }^{3}$ Department of Biotechnology and Molecular Biology, University of Opole, Opole, Poland; ${ }^{4}$ Department of Immunobiology of Bacteria, Institute \\ of Microbiology, Biotechnology and Immunology, University of Łódź, Łódź, Poland
}

\begin{abstract}
Most Proteus lipopolysaccharides (LPSs) contain uronic acids or their amides with different amino acids, which together with other negatively charged components account for the acidic character of such LPS molecules. Previous studies have shown the significance of an amide of galacturonic acid with lysine [D-GalA(L-Lys)] for serological specificity of $O$-antigens from few $P$. mirabilis strains. In this work, the immunodominant role of GalALys was indicated for the P. penneri 42 LPS core region. The studies also showed the serological identity of core oligosaccharides from $P$. penneri 42 (071), P. mirabilis 51/57 (O28) and R14/S1959 strains.
\end{abstract}

Key words: amide of galacturonic acid with lysine, anti-conjugate serum, core region, lipopolysaccharide, Proteus

Received: 31 October, 2013; revised: 25 February, 2014; accepted: 03 March, 2014; available on-line: 19 March, 2014

\section{INTRODUCTION}

Proteus bacteria, including $P$. mirabilis and $P$. penneri species, are human opportunistic pathogens causing different types of infections in preferable conditions, but the most frequent are urinary tract infections (UTIs) (Różalski et al., 2012). UTIs are often persistent and can lead to complications like pyelonephritis, as well as bladder or kidney stone formation. The latter process is mainly a result of the activity of urease, which catalyses the hydrolysis of urea to ammonia and carbon dioxide. The presence of ammonia leads to the elevation of $\mathrm{pH}$ and alkalization of urine, which in turn induces the precipitation of magnesium and calcium ions, and the formation of urinary stones, containing struvite and carbonate apatite. This phenomenon can be modified by the O-specific polysaccharide part of lipopolysaccharides (LPSs). Depending on the chemical structure of these polysaccharides and their ability to bind cations $\mathrm{Ca}^{2+}$ and $\mathrm{Mg}^{2+}$ the crystallization of struvite and carbonate apatite may be enhanced or inhibited. The negatively charged groups found in bacterial polysaccharides including LPSs play an important role in this process (Torzewska et al., 2003). It was found that negatively charged components like hexuronic acids, non-sugar carboxylic acids or phosphate groups are responsible for the acidic character of approximately $90 \%$ of Proteus sp. O-polysaccharides (OPSs) (Knirel et al., 2011). Hexuronic acids, including galacturonic acid (GalA) are often found in Proteus sp. OPSs, where they can be amidated with amino acids such as: L-alanine, L-serine and L-lysine (Lys; the most common) (Knirel et al., 2011). The GalA residue is present also in the Proteus LPS core region: in the proximal part of oligosaccharide - $\alpha$-linked GalA (in all structurally tested core oligosaccharides) and $\beta$-linked (mainly in P. mirabilis strains), as well as in the distal part — as an amide of GalA and Lys. The latter was demonstrated in one $P$. penneri strain and two $P$. mirabilis strains (Vinogradov et al., 2002; Vinogradov 2011). In the previously conducted studies GalALys appeared to play a crucial role in serological cross-reactions of $P$. mirabilis O28, S1959 LPSs and LPS from P. mirabilis R14/S1959 (a transient form of $P$. mirabilis S1959 containing T-antigen as a polysaccharide chain) with hetorologous polysaccharide-specific antisera (Radziejewska-Lebrecht et al. 1995; Bartodziejska et al., 1996). However, the above mentioned studies were focused on epitope characterization of the O-specific or T-specific (R14/S1959) polysaccharides and used polyclonal rabbit antisera against the $P$. mirabilis O28, S1959 and P. mirabilis R14/S1959 strains. These sera included a high level of polysaccharide-specific antibodies, which was revealed e.g. by the weak reaction of $P$. mirabilis R14/ S1959 antiserum with the core oligosaccharide fraction from homologous LPS and by the strong reaction with its T-polysaccharide fraction (Bartodziejska et al., 1996). The presence of the GalALys amide in the LPS core region of $P$. penneri 42 strain encouraged us to obtain the serum against the core oligosaccharide (OS) of the LPS of these bacteria (anti-conjugate serum) and check if the GalALys fragment is also important for the immunospecificity of the LPS core region.

\section{MATERIALS AND METHODS}

The set of bacterial strains. P. penneri $42(\mathrm{O} 71)$ is a clinical isolate from the patients from Toronto (Canada)

e-mail: agatapal@biol.uni.lodz.pl

*Presented at the 3-rd Workshop on Microbiology "MIKROBIOT 2013" in Łódź, Poland.

Abbreviations: Arap4N, 4-amino-4-deoxy-L-arabinopyranose; ELISA, enzyme-linked immunosorbent assay; GalA, galacturonic acid; GalALys, amide of GalA with the a-aminogroup of L-lysine; GalNAc, 2-acetamido-2-deoxy-D-galactose; Glc, glucose; GlcNGly, 2-deoxy-2-glycylamido-D-glucose; Hep, L-glycero-D-manno-heptose; DD-Hep, D-glycero-D-manno-heptose; Kdo, 3-deoxy-D-manno-oct2-ulosonic acid; MALDI-TOF, matrix-assisted laser-desorption/ionization time-of-flight mass spectrometry; LPS, lipopolysaccharide; OPS, O-poly-saccharide; OS, oligosaccharide; PEtn, 2-aminoethyl phosphate; UTIs, urinary tract infections. 
but its isolation source remains unknown. This strain together with the other 26 ones: $P$. penneri 1 (O72a), P. penneri 2 (O66), 4 (O72a,b), 7 (O61), 8 (O67), 11, 12 (O58), 13 (R form), 14 (O59), 15 (O52), 16 (O17), 17 (O8), 18 (O17), 19 (O64a,b,c), 22 (O63), 25 (O69), 26 (O31a), 27 (O64a,b,c), 28 (O31a,b), 31 (O19a,b), 34 (O65), 37 (R form), 39, 40 (O64a,b,d), 41 (O62), 44 (R form) were kindly provided by Professor D. J. Brenner, Center for Diseases Control and Prevention in Atlanta (USA). $P$. penneri 60 (O70), 63 (O68), 75 (O73a,b) and P. penneri 71 (O64a,c,e) strains were isolated from the urine of patients in hospitals in Lódź and Warsaw (Poland), respectively. The other strains were kindly provided: $P$. penneri 47 (O59) by Dr. E. Falsen, Department of Clinical Bacteriology, Göteborg (Sweden); P. penneri 77 (O65) and 93 (O67) by Dr. B. Senior, Department of Medical Microbiology in Dundee (UK); P. penneri 103 (O73a,c), 104 (O61), 107, 112 (O8) and P. penneri 124 (R form) by Dr. B. Holmes, Central Public Health Laboratory in London (UK); P. penneri 133 (O61) by Dr. G. Giammanco, Institute of Hygiene and Prevention Medicine in Catania (Italy). P. mirabilis strain G1 (O3a) was kindly provided by J. Gmeiner (Institute for Microbiology and Genetics, Darmstadt, Germany), P. mirabilis 51/57 (O28) comes from the Czech National Collection of Type Cultures, Institute of Epidemiology and Microbiology, Praque, Czech Republic. The above mentioned strains along with P. mirabilis S1959 (O3a,b) and its mutants: R110 and R14 (a transient-like form) belong to the collection of the Department of General Microbiology, University of Łódź (Poland), where they are stored in a glycerol mixture at $-80^{\circ} \mathrm{C}$. The bacteria were grown for $18 \mathrm{~h}$ under aerobic conditions in liquid nutrient broth containing $1 \%$ glucose (BTL, Poland) at $37^{\circ} \mathrm{C}$, killed with $1 \%$ phenol, centrifuged, washed twice with water and lyophilized to dried bacterial cells.

Lipopolysaccharide. The LPSs were extracted from dried bacterial cells by the phenol-water procedure according to the method by Westphal (Westphal \& Jann, 1965) and purified with aqueous 50\% trichloroacetic acid. Alkali-treated LPSs used for the sera adsorption were prepared as described in detail elsewhere (Palusiak et al., 2008).

After the degradation of $P$. penneri 42 LPS with $1.5 \%$ acetic acid $\left(100^{\circ} \mathrm{C}\right.$ for $\left.1 \mathrm{~h}\right)$ lipid $\mathrm{A}$ was separated from a water-soluble carbohydrate portion by centrifugation $\left(13000 \times g, 20 \mathrm{~min} ., 4^{\circ} \mathrm{C}\right)$. The sugar portion was then separated on a Bio-Gel P-10 column $(1.6 \times 100 \mathrm{~cm}$, equilibrated with $0.05 \mathrm{M}$ pyridine/acetic acid buffer, $\mathrm{pH}$ 5.6). Eluates were monitored with a Knauer differential refractometer. The fractions were eluted, freeze-dried and checked by matrix-assisted laser-desorption/ion- ization time-of-flight mass spectrometry (MALDI-TOF MS) run on a Kratos Kompact-SEQ instrument.

P. penneri 42 core oligosaccharide conjugate. The conjugation of the $P$. penneri 42 core oligosaccharide with diphtheria toxoid was performed by the method of $\mathrm{H}$. J. Jennings and C. Lugowski based on the reaction of reductive amination, which was described in detail elsewhere (Jennings \& Lugowski, 1981).

The $P$. penneri 42 anti-conjugate serum was gained by the immunization of New Zealand white rabbits according to the procedure described in detail elsewhere (Palusiak et al., 2008).

Serological assays. Purified LPS samples were tested with rabbit antisera in an enzyme-linked immunosorbent assay (ELISA), Western blot procedure after sodium dodecyl sulfate polyacrylamide gel electrophoresis with non-adsorbed antisera and/or antisera adsorbed with selected alkali-treated LPSs. All assays were performed as previously described (Sidorczyk et al., 2002; Palusiak et al., 2008).

\section{RESULTS AND DISCUSSION}

In this work, the serological specificity of the LPS core region from $P$. penneri 42 LPS was characterized. The first step of the study included the preparation of the $P$. penneri 42 diphtheria toxoid-core oligosaccharide conjugate, which was used as an immunogen in the process of obtaining the anti-conjugate serum. The serum, containing the antibodies specific to OS of homologous LPS, was checked in ELISA with a set of 45 Proteus LPS preparations. These antigens were selected for the study on the grounds of previously conducted investigations and come from strains representing many Proteus O-serogroups. The tested antiserum reacted only with five LPSs $[P$. penneri 42 (O71), P. mirabilis 51/57 (O28), G1 (O3a), S1959 (O3a,b) and P. mirabilis R14/ S1959]. As shown in Table 1, only one LPS, P. mirabilis $\mathrm{R} 14 / \mathrm{S} 1959$, reacted to the same titer as $P$. penneri 42 LPS, P. mirabilis 51/57 (O28) LPS reacted with heterologous antibodies similarly to the homologous antigen, meanwhile the remaining two LPSs bound the immunoglobulins much weaker. In the Western blot technique, after the separation of LPS samples in sodium dodecyl sulfate polyacrylamide gel electrophoresis, P. penneri 42 anti-conjugate serum recognized fast migrating bands (restricted to core-lipid A molecules) of P. penneri 42, P. mirabilis 51/57 and R14/S1959 LPSs, which core regions seem to be identical. Moreover, the tested serum bound to slow migrating bands (corresponding to high-molecular-mass LPS species containing a core-lipid A moiety substituted with O-polysaccharide chains) of P. mirabilis

Table 1. ELISA data of the reactivity of Proteus sp. LPSs with adsorbed anti-conjugate serum against $P$. penneri 42 strain ${ }^{\mathrm{a}, \mathrm{b}}$ aNon-adsorbed antiserum was used as control; bData for homologous LPS are bolded

\begin{tabular}{|c|c|c|c|c|c|}
\hline \multirow{3}{*}{$\begin{array}{l}\text { P. penneri } 42 \\
\text { anti-conjugate serum }\end{array}$} & \multicolumn{5}{|c|}{ Reciprocal titer of adsorbed antiserum for LPS from strains } \\
\hline & \multirow{2}{*}{$\begin{array}{l}\text { P. penneri } \\
42\end{array}$} & \multicolumn{4}{|c|}{ P. mirabilis } \\
\hline & & $51 / 57$ & G1 & S1959 & R14/S1959 \\
\hline Control & 8.000 & 4.000 & 2.000 & 2.000 & 8.000 \\
\hline P. penneri 42 & $<500$ & $<500$ & $<500$ & $<500$ & $<500$ \\
\hline P. mirabilis $51 / 57$ & $<500$ & $<500$ & $<500$ & $<500$ & $<500$ \\
\hline P. mirabilis G1 & 4.000 & 2.000 & $<500$ & $<500$ & 4.000 \\
\hline P. mirabilis S1959 & 4.000 & 2.000 & $<500$ & $<500$ & 4.000 \\
\hline P. mirabilis R14/S1959 & $<500$ & $<500$ & $<500$ & $<500$ & $<500$ \\
\hline
\end{tabular}




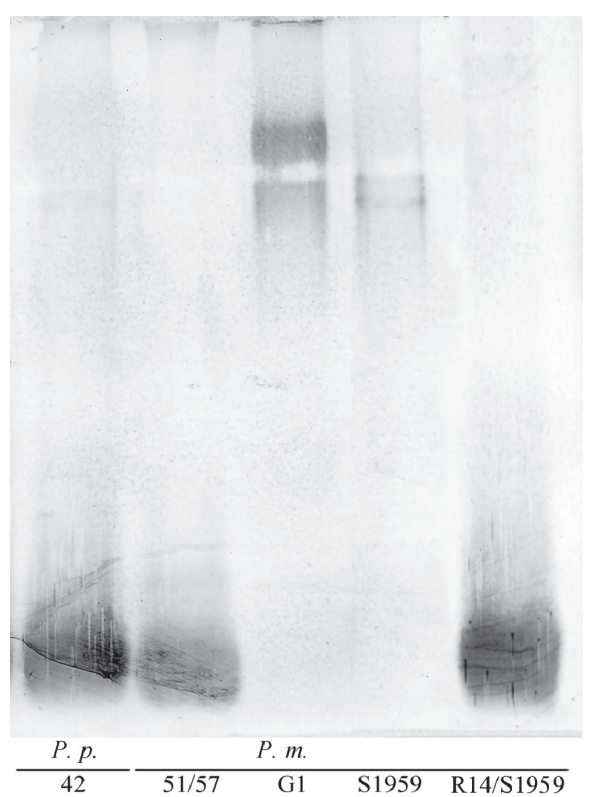

Figure 1. Western blot of Proteus sp. LPSs with serum against the core oligosaccharide of the $P$. penneri 42-diptheria toxoid conjugate.

G1 and S1959 LPS preparations. Similarly to the results obtained in ELISA (Table 1), the last two reactions were weaker than those observed for the other tested LPSs. To confirm the similarities in serological specificity of the used antigens the adsorption procedure of the antiserum by the tested LPS preparations was performed. The reactivity of the adsorbed antiserum (compared to the non-adsorbed one) was checked in ELISA with all used LPSs. Adsorption of antiserum by alkali-treated LPSs of P. penneri 42, P. mirabilis $51 / 57$ and R14/S1959 strains completely abolished the serum reactions with all used antigens (Table 1), which confirmed the identical binding patterns observed for these three LPSs in the Western blot technique (Fig. 1). After the serum adsorption by $P$. mirabilis G1 and S1959 LPSs, there was no reactivity in these systems, whereas the reactivity titers for $P$. penneri 42, P. mirabilis 51/57 and P. mirabilis R14/S1959 LPSs were slightly lower compared to the control systems.

To summarize, the core oligosaccharides of $P$. penneri 42 and $P$. mirabilis 51/57 LPSs have a common major epitope responsible for the strong reactions of these LPSs with homologous and heterologous antibodies, respectively. Such outcomes stay in agreement with the previously published results of chemical analysis, which showed the structural identity of the LPS core regions from $P$. penneri 42 and $P$. mirabilis $51 / 57$ strains (Vinogradov et al., 2002). P. mirabilis G1 and S1959 OPSs contain a minor common epitope, which is also localized in the LPS core regions of $P$. penneri 42 and $P$. mirabilis 51/57 LPSs.

Interestingly, these studies revealed that there is another Proteus LPS (P. mirabilis R14/S1959), which probably possesses a major epitope common with the OS of $P$. penneri 42 and probably P. mirabilis 51/57 LPSs. It was found by the reactivity of the $P$. penneri 42 anti-conjugate serum with R14/S1959 LPS, which was identical in the reactions observed in ELISA and in Western blot for $P$. penneri 42 LPS. What is most important, this LPS used for the adsorption of the $P$. penneri 42 anti-conjugate serum abolished the reactions with all tested LPSs ( $P$. penneri 42, P. mirabilis 51/57, G1 and S1959), which is the best proof of the serological identity of the OS from P. penneri 42 and P. mirabilis R14/S1959 LPSs (Table 1). The previous serological studies using antiserum against P. mirabilis O28 strain suggested only the presence of a common epitope (probably GalALys) in the LPS of $P$. mirabilis $\mathrm{O} 28$ and the core region of $P$. mirabilis R14/ S1959 LPS, but they did not reveal the identical serospecificity of the core regions from these two LPSs (Radzie-

\section{a. . penneri $42(\mathrm{O} 71)$ and $P$. mirabilis $51 / 57(\mathrm{O} 28) \mathrm{R}^{3}=\mathrm{H}$ (Vinogradov et al., 2002)}

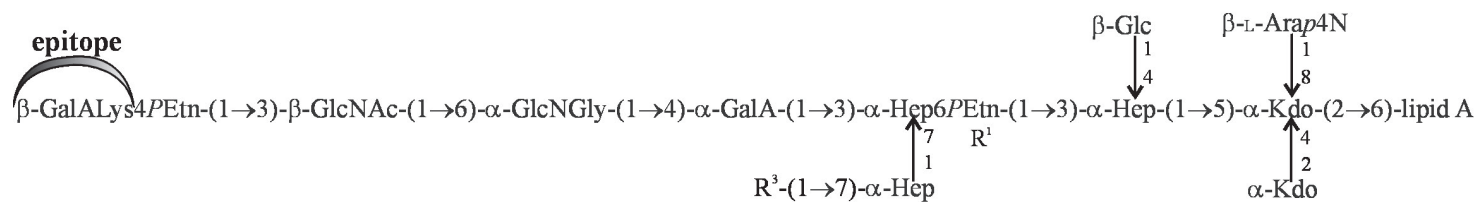

b. P. mirabilis $\mathrm{S} 1959$ (O3a,b) (Knirel et al., 2011)

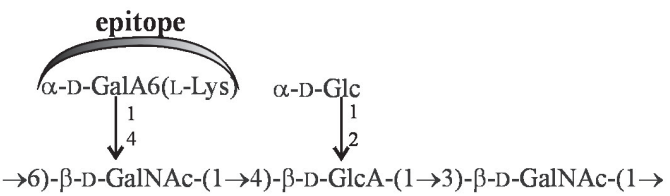

c. P. mirabilis $\mathrm{G} 1(\mathrm{O} 3 \mathrm{a})$ (Sidorczyk et al., 2002)

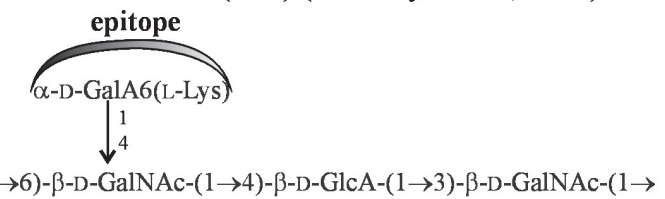

Figure 2. Structures of: the core region of $P$. penneri 42 and $P$. mirabilis $51 / 57$ LPS (a); O-polysaccharide of $P$. mirabilis S1959 (b) and P. mirabilis $\mathrm{G} 1$ (c)

A common epitope is indicated by arcs. Arap4N, 4-amino-4-deoxy-L-arabinopyranose; GalA, galacturonic acid; GalALys, amide of D-GalA with the a-aminogroup of L-lysine; GalNAc, 2-acetamido-2-deoxy-D-galactose; Glc, glucose; GlcNGly, 2-deoxy-2-glycylamido-D-glucose; Hep, L-glycero-D-manno-heptose; DD-Hep, D-glycero-D-manno-heptose; Kdo, 3-deoxy-v-manno-oct-2-ulosonic acid; PEtn, 2-aminoethyl phosphate. 
jewska-Lebrecht et al., 1995; Bartodziejska et al., 1996). It is worth mentioning that although P. mirabilis R14/ S1959 strain is a transient mutant of S1959 strain, its polysaccharide T-antigen (similar to the T1 chain of Salmonella Friedenau LPS) is characterized by the structure completely different from the structure of the O-antigen of its parental strain. At the same time, the T-antigen of $P$. mirabilis R14/S1959 is identical in its structure with the OPS of P. penneri 42 LPS (Bartodziejska et al., 1996; Knirel et al., 2011). Combining this fact with the results of the serological studies, showing the identity of serological specificity of the core regions from $P$. penneri 42 and P. mirabilis R14/S1959 LPSs, it seems to be probable that both LPSs have much in common not only in their polysaccharide parts but also in the core regions. It is interesting since such huge structural and/or serological similarities between LPSs of the strains from two different species are rather uncommon.

Comparing the structures of OS from $P$. penneri 42 and $P$. mirabilis 51/57 LPSs and of OPS from P. mirabilis G1 and S1959 LPSs (the structures differ only in the lateral D-Glc residue present in P. mirabilis S1959 LPS), it has been revealed that they all share an amide of galacturonic acid with lysine as a distal component of the core region of the first mentioned LPSs ( $P$. penneri 42 and $P$. mirabilis 51/57) and as a lateral branch in OPS of the others (Fig. 2) (Knirel et al., 2011; Sidorczyk et al., 2002; Radziejewska-Lebrecht et al., 1995; Vinogradov et al., 2002). These fragments differ only in their conformation ( $\beta$-linked in P. penneri 42 and P. mirabilis $51 / 57$ core regions and $\alpha$-linked in $P$. mirabilis G1 and S1959 OPSs). As showed by Radziejewska-Lebrecht et al. (1995), using the synthetic glycopolymers containing amides of D-GalA with different amino acids, including lysine, the immunodominant part of the mentioned epitope is probably an $\mathrm{NH}_{2}\left(\mathrm{CH}_{2}\right)_{4}$ group of lysine rather than the whole residue. According to these data, the $\alpha$ or $\beta$ type of linkage may not play an important role in the immunospecificity of the amide.

There are two reasons supporting the hypothesis of the GalALys domination in the specificity of the $P$. penneri 42 LPS core region. Firstly, $P$. penneri 42 anti conjugate serum reacted in ELISA only with LPSs possessing the mentioned amide in their structures. Secondly, the other studies indicated that the majority of antibodies in the core-specific sera recognize the most distal fragments of the LPS core regions (Palusiak et al., 2008; Palusiak \& Sidorczyk, 2010), and the GalALys amide represents this type of residues.

Indicating such immunogenic epitopes as GalALys, common not only for the LPS core regions but also for its O-polysaccharides from different species, should be found important because of the potential application of such fragments as synthetic antigens used for obtaining an appropriate protective serum.

\section{Acknowledgements}

This work was supported by the Ministry of Science and Higher Education (Poland), grant No. N 401001 $31 / 0009$.

\section{REFERENCES}

Bartodziejska B, Radziejewska-Lebrecht J, Lipinska M, Knirel YA, Kononov LO, Chernyak AY, Mayer H, Rozalski A (1996) Structural and immunochemical studies on the lipopolysaccharide of the 'T-antigen'-containing mutant Proteus mirabilis R14/1959. FEMS Immunol Med Microbiol 13: 113-121.

Jennings HJ, Lugowski C (1981) Immunochemistry of groups A, B, and $\mathrm{C}$ meningococcal polysaccharide-tetanus toxoid conjugates. $J$ Immunol 127: 1011-1018.

Knirel YA, Perepelov AV, Kondakova AN, Senchenkova SN, Sidorczyk Z, Rozalski A, Kaca W (2011) Structure and serology of O-antigens as the basis for classification of Proteus strains. Innate Immunity 17: 70-96.

Palusiak A, Dzieciątkowska M, Sidorczyk Z (2008) Application of two different kinds of sera against the Proteus penneri lipopolysaccharide core region in search of epitopes determining cross-reactions with antibodies. Arch Immunol Ther Exp 56: 135-140.

Palusiak A, Sidorczyk Z (2010) Characterization of epitope specificity of Proteus penneri 7 lipopolysaccharide core region. Acta Biochim Polon 57: 529-532.

Radziejewska-Lebrecht J, Shashkov AS, Vinogradov EV, Grosskurth H, Bartodziejska B, Rozalski A, Kaca W, Kononov LO, Chernyak AY, Mayer H, Knirel YA, Kochetkov NK (1995) Structure and epitope characterisation of the O-specific polysaccharide of Proteus mirabilis O28 containing amides of D-galacturonic acid with L-serine and L-lysine. Eur J Biochem 230: 705-712.

Różalski A, Torzewska A, Moryl M, Kwil I, Maszewska A, Ostrowska K, Drzewiecka D, Zabłotni A, Palusiak A, Siwińska M, Stączek P (2012) Proteus sp. - an opportunistic bacterial pathogen — classification, swarming growth, clinical significance and virulence factors. Folia Biologica et Oecologica 8: 1-17.

Sidorczyk Z, Zych K, Toukach FV, Arbatsky NP, Zabłotni A, Shashkov AS, Knirel YA (2002) Structure of the O-polysaccharide and classification of Proteus mirabilis strain G1 in Proteus serogroup O3. Eur J Biochem 269: 1406-1412.

Torzewska A, Stączek P, Różalski A (2003) Crystallization of urine mineral components may depend on the chemical nature of Proteus endotoxin polysaccharides. J Med Microbiol 52: 471-477.

Vinogradov E (2011) Structure of the core part of the lipopolysaccharide from Proteus mirabilis genomic strain HI4320. Biochemistry (Moscow) 76: 803-807.

Vinogradov EV, Sidorczyk Z, Knirel YA (2002) Structure of the lipopolysaccharide core region of the bacteria of the genus Proteus. Aust J Chem 55: 61-67.

Westphal O, Jann K (1965) Bacterial lipopolysaccharides. Extraction with phenol-water and further applications of the procedure. Methods Carbohydr Chem 5: 83-91. 